\title{
Quasi-distributed Vibration Sensing Based on Weak Reflectors and STFT Demodulation
}

\author{
Daniel Leandro*, Mengshi Zhu*, Manuel Lopez-Amo and Hideaki Murayama
}

\begin{abstract}
In this work, a new system is proposed for quasidistributed vibration sensing based on the Doppler effect. The setup employs an optical frequency domain reflectometer (OFDR) and an array of weak reflectors. The short-time Fourier transform (STFT) is employed to obtain the spectrogram, then the demodulation process detects the frequency variation and compensates the accumulated response. In this manner, events occurring in the fiber segment between neighboring reflectors can be measured. The theoretical development of the approach is presented and demonstrated, evidencing the velocimeter operation of the technique. The setup has been proved in several tests under different perturbation conditions, evaluating the limitations and capabilities. This solution presents many advantages such as the ability to measure arbitrary perturbations, the linear response, high spatial-resolution, simplicity, high configurability or the low repetition rate required to detect kilohertz-frequency vibrations.
\end{abstract}

Index Terms - Optical fiber, Velocity measurement, Reflectometry, Short-time Fourier transform.

\section{INTRODUCTION}

FIBER-OPTIC techniques on quasi-distributed and distributed vibration/acoustic sensing (DVS/DAS) are attracting a lot of attention for their excellent potential in oil and gas pipeline applications, seismic detection, intrusion systems, etc. [1-4]. Among the reported techniques, fully distributed DVS with kilometers' measurement distance have attracted lots of interest in applications involving large-scale structures such as pipelines, or bridges. However, there are medium-scale applications where the distance required is in the order of hundreds of meters. For example, in aeronautics, elements of interest such as fuselage or wings require sensing lengths within $100 \mathrm{~m}$. In this manner, systems with shorter sensing distance and higher spatial resolution are more efficient. [5-7] Currently, in this scale, one broadly commercialized solution is optical frequency domain reflectometry (OFDR) whose typical sensing length is from ten to hundreds of meters. However, to the best

Manuscript received CCC 2020; revised XX 2020; accepted XX 2020. Date of publication XX 2020; date of current version XX, 2020. This work was supported by a Fundación La Caixa postdoc and Talent Grant, the project AEI TEC-2016-76021-C2-1 and the FEDER funds (Corresponding author: Mengshi Zhu).

Daniel Leandro and Manuel López-Amo are with the Department of Electrical, Electronic and Communication Engineering, Universidad Publica de Navarra and the Institute of Smart Cities ISC, Campus de Arrosadia, Pamplona 31006, Spain (e-mail: daniel.leandro@unavarra.es, mla@unavarra.es). of our knowledge, the commercial products are usually for static or quasi-static applications where the repetition rate is lower than $10 \mathrm{~Hz}$, limiting its application in vibration sensing. Advanced achievements have been reported utilizing highspeed OFDRs in the laboratory [8].

A common approach to measure vibration using fiber optics is by measuring strain changes in the fiber at high frequency. Consequently, the maximum frequency detected is limited by the repetition rate of the interrogator (by the Nyquist theorem). However, in order to detect hundreds-thousands kilohertz, it is usually required high-end (costly) equipment, especially in OFDR setups. To further enhance the potential of OFDR systems, this work focuses on the cost-effective realization of a low-repetition rate OFDR for high-frequency measurements exploiting the Doppler effect in a quasi-distributed configuration.

In this case, the quasi-distributed concept using a weakreflectors array, as fully-distributed, monitors the whole fiber length, but defining the spatial-resolution discretely using reflectors. An inherent advantage of using weak reflector array is the higher signal-to-noise ratio with respect to Rayleigh backscattering and the ease of the position synchronization after installation. Some works have been already published including a weak-reflector array for vibration/acoustic sensing using OFDR. In [9], a double interrogation technique with a sinusoidally-modulated laser is employed to detect acoustic waves. In our recent publication [10], a low-repetition rate OFDR demodulation technique using weak reflectors was validated for 30 sections with a spatial resolution $\sim 15-20 \mathrm{~cm}$. In the approach, up to 30 kilohertz vibration signals were detected by a Fourier analysis and an iterative side peak compensation technique. However, this solution suffered important downsides that limited its use in practical applications. For example, it cannot accurately detect high-velocity events (it suffers from overmodulation) and particularly, it is not able to reconstruct arbitrary (non-periodic) signals.

It this work, we propose, evaluate and demonstrate a new demodulation technique based on the short time Fourier

Mengshi Zhu was with the Graduate School of Engineering, University of Tokyo, 7-3-1 Hongo, Bunkyo-ku, Tokyo 113-8654, Japan and is now with the Optical Technology R\&D Center, Fujikura Ltd., 1440, Mutsuzaki, Sakura, Chiba 285-8550, Japan (e-mail: mengshi.zhu@jp.fujikura.com).

Hideaki Murayama is with the Graduate School of Frontier Sciences, University of Tokyo, 5-1-5 Kashiwanoha, Kashiwa-shi, Chiba 277-8561, Japan (e-mail: murayama@edu.k.u-tokyo.ac.jp).

"These authors contributed equally to this work. Digital Object Identifier XXX 
2

JLT-26059-2020.R1

transform (STFT) and image processing, to measure quasidistributed vibration using a low-repetition rate OFDR and inline reflectors. This novel approach is based on the principle of in-line velocimeters, and it can measure high amplitude and arbitrary-waveform events. The sensing principle is theoretically explained and verified by experiments. According to the results, the proposed technique is highly configurable and allows the measurement of arbitrary vibrations and the reconstruction of the waveform for spatial resolutions in the centimeter-order. Since no reference or calibration are required, this quasi-DVS is able to perform absolute measurements without suffering any amplitude uncertainty (common in DVS) due to effects such as phase-wrapping.

\section{PRINCIPLE OF OPERATION}

The proposed technique is based on an OFDR, the sensing arm of which consists of $N$ in-line weak reflectors (WRs). For the sake of clarity in the theoretical analysis, a simplified schematic of the sensing system is illustrated in Fig. 1.

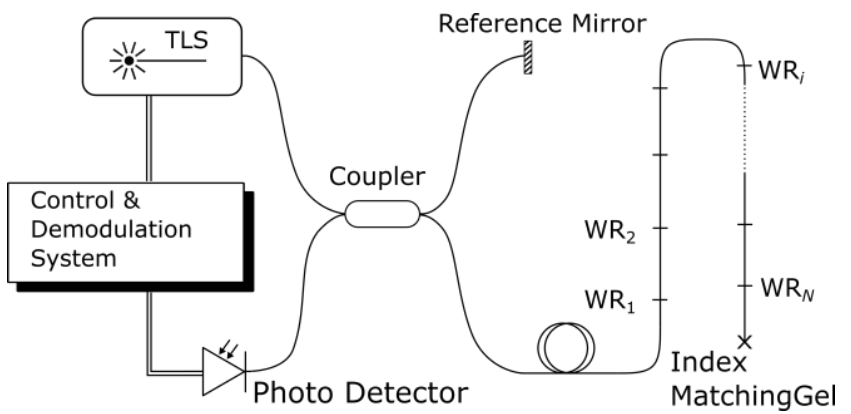

Fig. 1. Simplified schematic of the sensing system.

The tunable laser source sweeps at the speed of $\gamma$ in the frequency domain. Therefore, by ignoring the fluctuating phase, the reflected light wave from the reference arm in the interferometer can be simply expressed as

$$
E_{\mathrm{ref}}=A_{\mathrm{ref}} \exp \left[j\left(2 \pi f_{0} t+\pi \gamma t^{2}+C_{0}\right)\right]
$$

where $A_{\text {ref }}$ is the amplitude, $f_{0}$ is the initial frequency of the injected light, $t$ is the time and $C_{0}$ is the initial optical phase. Assuming that there is a local perturbation in the segment between $W R_{i-1}$ and $W R_{i}$, the variation of the path, which is the function of time, can be expressed as

$$
l_{i}=D \sin \left(2 \pi f_{\mathrm{v}} t+\phi_{\mathrm{v}}\right)+l_{i, 0},
$$

where $D$ is the amplitude of the vibration, $f_{\mathrm{v}}$ is the vibration frequency, $\phi_{\mathrm{v}}$ is the corresponding phase, and $l_{i, 0}$ is the initial path length. Please note that according to the theorem of Fourier series, any arbitrary signal can be expanded to a summation of sinusoidal functions. Thus, to simplify the mathematical expressions, a single sinusoidal function is used. Any arbitrary event-waveform can be detected similarly. The speed of the path change can be obtained by

$$
V_{i}=\frac{d l_{i}}{d t}=2 \pi D f_{\mathrm{v}} \cos \left(2 \pi f_{\mathrm{v}} t+\phi_{\mathrm{v}}\right)
$$

Assume that the effective refractive index of the optical fiber is $n_{\text {eff. }}$ Considering the Doppler effect in optical fiber $[11,12]$ that induces an optical frequency shift of value $\left(n_{\text {eff }} V_{i} f_{0}\right) / c$ and the fact that the speed range of the TLS is limited to several nanometers per second, the corresponding reflected light can be derived as

$$
E_{i}=A_{i} \exp \left\{j\left[2 \pi \int_{0}^{t-p_{i}}\left(f_{0}+\gamma t+\frac{n_{\mathrm{eff}} V_{i}}{c} f_{0}\right) d t+C_{1, i}\right]\right\},
$$

where $p_{i}=2 n_{\mathrm{eff} z} / c$ is the round-trip time delay between the reference reflector and $W R_{i}$ at the position, $z_{i}$. And $C_{1, i}$ is the corresponding optical phase. Consequently, the interference beat signal from the reference and sensing arms can be expressed by

$$
\begin{aligned}
I_{i} & =\left(E_{\mathrm{ref}}+E_{i}\right)\left(E_{\mathrm{ref}}+E_{i}\right)^{*} \\
& \propto A_{\mathrm{ref}} A_{i} \exp \left(j\left\{\begin{array}{c}
2 \pi f_{0} p_{i}+2 \pi \gamma p_{i} t-\pi \gamma p_{i}^{2} \\
-\frac{n_{\mathrm{eff}} D f_{0}}{c} \sin \left[2 \pi f_{\mathrm{v}}\left(t-p_{i}\right)\right]
\end{array}\right\}\right) .
\end{aligned}
$$

If the STFT is applied to (5), the obtained spectrogram can be approximately expressed as

$$
W_{i}=\int_{-\infty}^{\infty} I_{i} \operatorname{rect}\left(\frac{t-\tau}{w}\right) e^{-j \omega t} d t,
$$

where $\omega$ is the angular frequency, $\tau$ is the central time of the rectangular window function, and $w$ is its time-width. If the width of the rectangular function is small, the expression can be further derived to be

$$
\begin{aligned}
W_{i} & \approx \int_{-\infty}^{\infty} A_{\text {ref }} A_{i} \exp \left(j \left\{2 \pi f_{0} p_{i}-\pi \gamma p_{i}^{2}+2 \pi \gamma p_{i} t\right.\right. \\
& \left.\left.+\frac{2 \pi n_{\mathrm{eff}} D f_{0} f_{\mathrm{v}}}{c} \cos \left[2 \pi f_{\mathrm{v}}\left(\tau-p_{i}\right)\right](t-\tau+w / 2)\right\}\right) \\
& \operatorname{rect}\left(\frac{t-\tau}{w}\right) e^{-j \omega t} d t \\
& \propto \delta\left(\omega-2 \pi \gamma p_{i}-\frac{2 \pi n_{\mathrm{eff}} D f_{0} f_{\mathrm{v}}}{c} \cos \left[2 \pi f_{\mathrm{v}}\left(\tau-p_{i}\right)\right]\right) .
\end{aligned}
$$

Setting $\omega=4 \pi \cdot n_{\text {eff }} \cdot \gamma \cdot z / c$, and considering $p_{i}=2 n_{\text {eff }} z_{i} / c$, it can be found that

$$
W_{i} \propto \delta\left(z-z_{i}-\frac{D f_{0} f_{\mathrm{v}}}{2 \gamma} \cos \left[2 \pi f_{\mathrm{v}}\left(\tau-p_{i}\right)\right]\right) .
$$

The high energy distribution in the time spatial domain, referring to $W_{i}=1$, can be extracted to be 


$$
z=z_{i}+\frac{D f_{0} f_{\mathrm{v}}}{2 \gamma} \cos \left[2 \pi f_{\mathrm{v}}\left(\tau-p_{i}\right)\right] .
$$

If we eliminate the DC signal $z$, that corresponds to the initial position of the $i$-th reflector, the waveform induced by the perturbation can be obtained as

$$
u_{i}=\frac{D f_{0} f_{\mathrm{v}}}{2 \gamma} \cos \left[2 \pi f_{\mathrm{v}}\left(\tau-p_{i}\right)\right] .
$$

In a linear sensing channel, perturbations accumulate and propagate until reflected. That implies that when several perturbations occur in the path before $W R_{i}$, the obtained waveform will be the linear sum of them. Note that higher order reflections that can cause crosstalk are in practice negligible due to the use of weak reflectors and an amplitude-independent detection technique. The obtained waveform can be expressed then as:

$$
U_{i}=\sum_{m=1}^{i} u_{m}(\tau)
$$

where $U_{i}$ is the cumulated perturbations before $W R_{i}$. Thus, the local perturbation can be demodulated just by subtracting the previous accumulated signal after the initial position, as described by

$$
u_{i}=\left\{\begin{array}{cr}
U_{1} & , i=1, \\
U_{i}-U_{i-1}, & i>1 .
\end{array}\right.
$$

Therefore, the perturbations signals of multiple events can be unambiguously measured by applying the STFT to the interferogram obtained using a simple OFDR and an array of weak reflectors. Since the phase change is accumulative along the fiber, the perturbation affecting each section can be iteratively obtained by subtracting the perturbations of the previous sections.

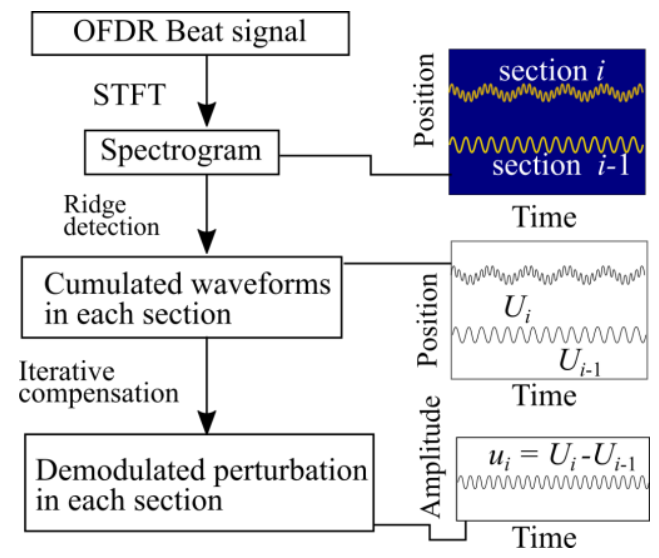

Fig. 2 Schematic diagram of the demodulation process.

To validate the proof-of-concept, a ridge detection is used to get the cumulated waveform at each section after the STFT as depicted in Fig. 2. Then, the waveforms are compensated iteratively so the independent perturbation in each section is obtained.

\section{EXPERIMENTAL RESULTS}

\section{A. Experimental setup}

Several experiments have been carried out to evaluate the demodulation and practical performance of the approach. In these experiments, different number of in-line WRs were placed in the sensing arm of an OFDR to define the sensing segments. The schematic of the experimental setup used is illustrated in Fig. 3. It combines two interferometers. The auxiliary one is included to generate a clock signal to trigger the data acquisition of the main one. Accordingly, the sampling rate of the beat signal is expressed as

$$
f_{\mathrm{s}}=\frac{2 n_{\mathrm{eff}} L_{\mathrm{R}} \gamma}{c}
$$

where $L_{R}$ is the path difference between reflector R1 and R2. The detailed principle of this OFDR configuration have been explained in depth in $[13,14]$.

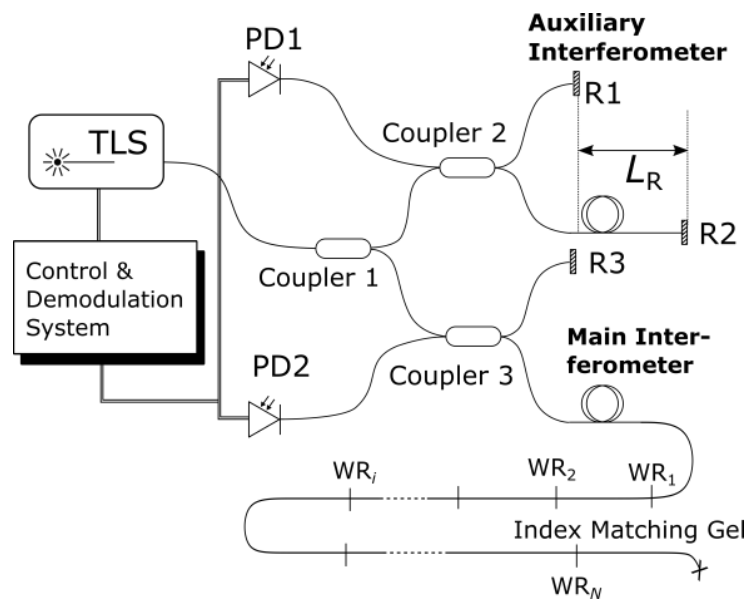

Fig. 3. Schematic of the OFDR-based sensing system. PD1,2: photo detector 1,2 ; $\mathrm{WR}_{1 \sim N}$ : reflector $1 \sim N$.

\section{B. Simple and multiple-vibration measurements}

A first set of measurements was performed to show the feasibility of the demodulation technique. To do this, an array of 10 weak-reflectors (FC/PC interconnections) separated $\sim 1 \mathrm{~m}$ was located in a $10 \mathrm{~m}$-section of standard single-mode fiber. The intensity distribution of the WRs in static condition in spatial domain is shown in Fig. 4. The area before $\mathrm{WR}_{1}$ is identified as Segment $1\left(\mathrm{Seg}_{1}\right)$. The area between $\mathrm{WR}_{i-1}$ and $\mathrm{WR}_{i}$ is defined as $\mathrm{Seg}_{i}$. The reflectors have nearly similar reflectivities which are all above the Rayleigh floor. Then, vibrations in 3 conditions are applied using piezo-electric ceramic transducers for each corresponding OFDR setups, as given in Table 1. 


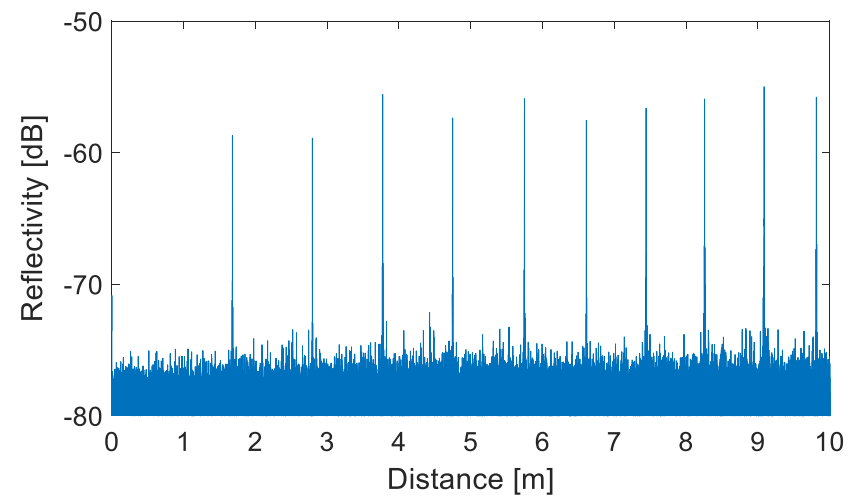

Fig. 4. Intensity distribution of 10 in-line weak reflectors.

Table 1. Vibration conditions and measurement setups.

\begin{tabular}{llllll}
\hline \hline Test & $\begin{array}{l}\text { Vibration } \\
\text { type }\end{array}$ & $\begin{array}{l}\text { Vibrated } \\
\text { segment }\end{array}$ & $\begin{array}{l}\text { Vibration } \\
\text { frequency } \\
{[\mathrm{Hz}]}\end{array}$ & $\begin{array}{l}\text { TLS } \\
\text { tuning } \\
\text { speed } \\
{[\mathrm{THz} / \mathrm{s}]}\end{array}$ & $\begin{array}{l}\text { Wavel. } \\
\text { range } \\
{[\mathrm{nm}]}\end{array}$ \\
\hline 1 & Sinusoidal & 3 & 300 & 2.6 & 20 \\
2 & Sinusoidal & 9 & 100 & 2.6 & 20 \\
3 & Sinusoidal & $3 \& 9$ & $300 \& 100$ & 2.6 & 20 \\
\hline
\end{tabular}

In the first two tests, a single sinusoidal perturbation of frequencies 300 and $100 \mathrm{~Hz}$ is applied to segments 3 and 9 respectively. In order to extract the perturbation occurring at each segment, a classic ridge detection (Matlab built-in), was performed to the spectrogram. Then the perturbation at each section is obtained by subtracting the accumulated signal at the previous section as described in (12). The demodulated waveforms depicted in Fig. 5 show a clear sinusoidal pattern without crosstalk from adjacent segments.
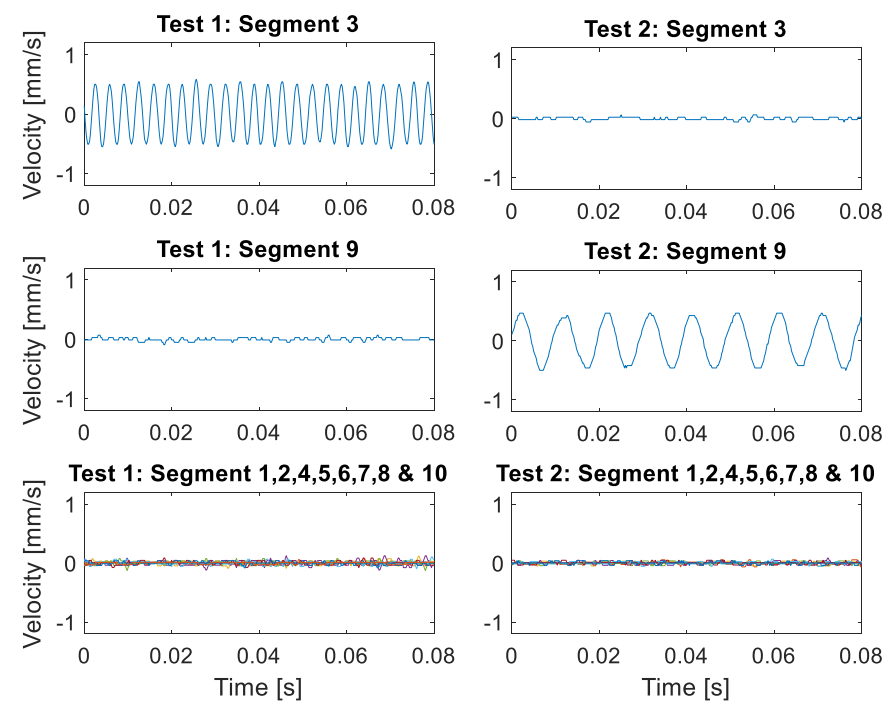

Fig. 5. Results for test 1 and test 2; vibrations of 300 and $100 \mathrm{~Hz}$ applied to segment 3 and 9 respectively.
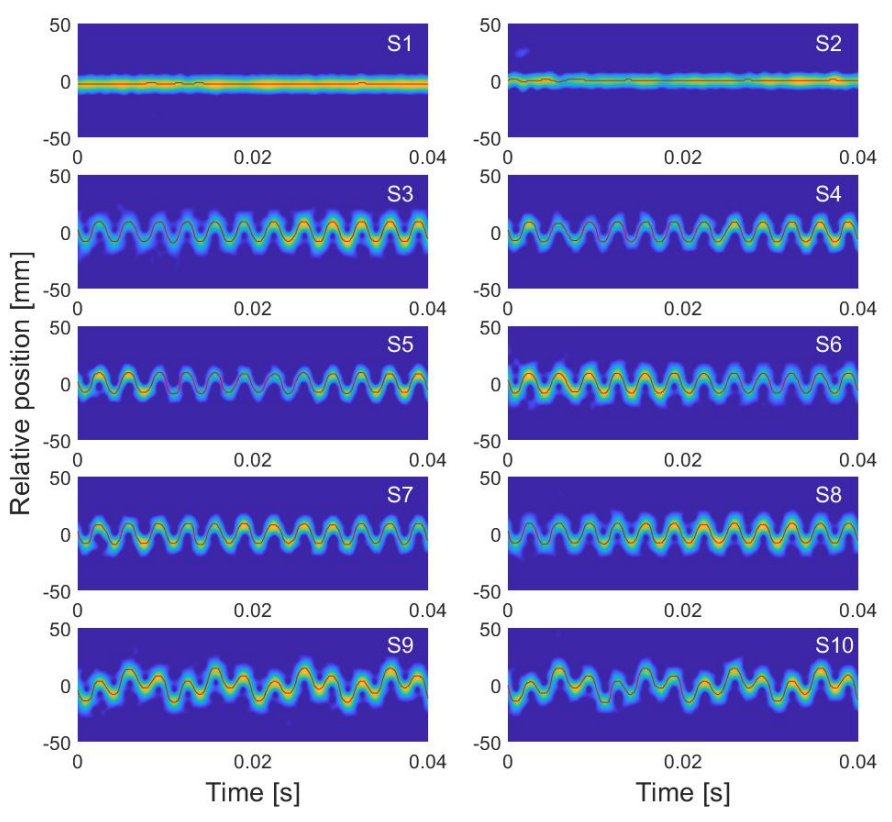

Fig. 6. Spectrograms obtained for every section in test 3 . Superimposed: signal retrieved after ridge detection.
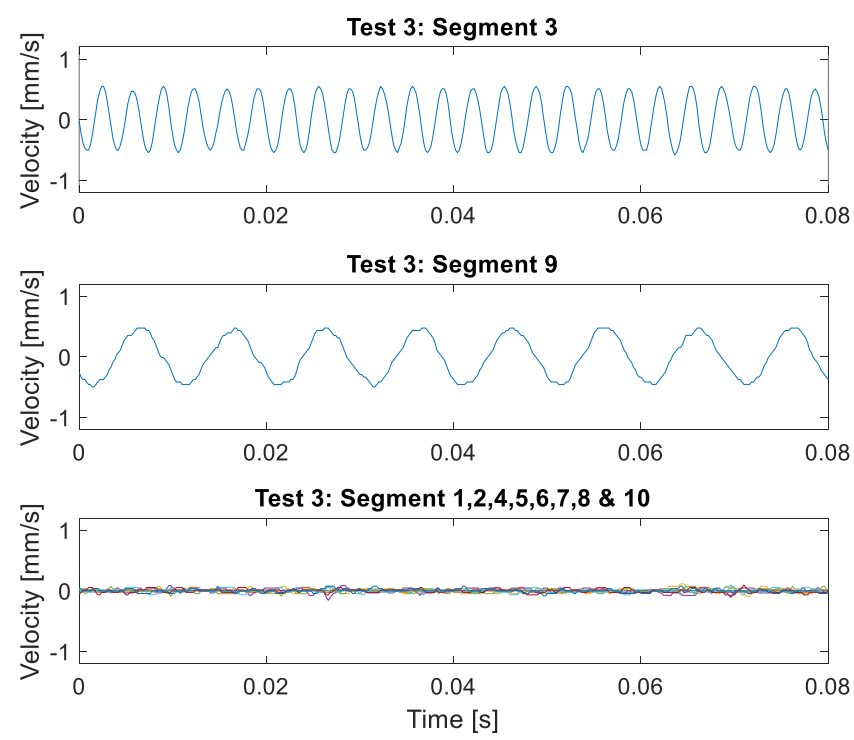

Fig. 7. Demodulated waveforms for test 3.

In test 3, two different sinusoidal perturbations of 300 and 100 $\mathrm{Hz}$ are applied to segments 3 and 9 simultaneously to verify the appropriate demodulation process for multiple events in the fiber. Figure 6 depicts the spectrogram of every section (clipped to avoid blank gaps between sections) and signal obtained after the ridge detection. The accumulative nature of the changes induced by the vibrations is evidenced there. In this manner, Segments 1 and 2 remained stable; Segment 3 reflects the first $100 \mathrm{~Hz}$ sinusoidal vibration, that it is mirrored in the following segments $(4,5,6,7,8)$; Segment 9 shows the addition of the vibration applied in this segment plus the already carried from the previous segment; Segment 10, with no specific 
Finally, after the ridge-detection and the compensation, Fig. 7 shows the results of the demodulated perturbations for all the segments, evidencing the crosstalk-free identification of the 300 and $100 \mathrm{~Hz}$ sinusoidal signals applied to Segment 3 and 9.

\section{Validation as velocimeter}

As explained in the sensing principle, the system is a quasidistributed velocimeter based on the Doppler effect. That implies that the demodulated waveform represents the derivative of the displacement signal applied to the fiber with respect to time; as expressed in (10) for a sinusoidal signal. However, this demodulation techniques also allows arbitrary waveforms to be detected in the same manner. Thus, a generic expression can be inferred for an arbitrary displacement signal $l_{i}(\tau)$ which derivative with respect to time is $V_{i}(\tau)$ :

$$
u_{i}=\frac{f_{0}}{4 \pi \gamma} \cdot V_{i}\left(\tau-p_{i}\right)=\frac{f_{0}}{4 \pi \gamma} \cdot \frac{d}{d \tau} l_{i}\left(\tau-p_{i}\right)
$$

Due to the unknown initial phase of the generated vibration, sinusoidal waves are not appropriate to prove this relationship. Thus, for verification, a square wave was applied to only one reflector of the sensing arm. Fig. 8 (a) shows the measured displacement applied using a reference displacement sensor whereas Fig. 8(b) depicts its derivative with respect to time. The obtained spectrogram is shown in Fig. 8(c). It can be seen that the pattern represented in the spectrogram agrees with the $1^{\text {st }}$ order derivative of the applied wave, evidencing the velocimeter nature of the system. The demodulated waveform is shown in Fig.8(d).
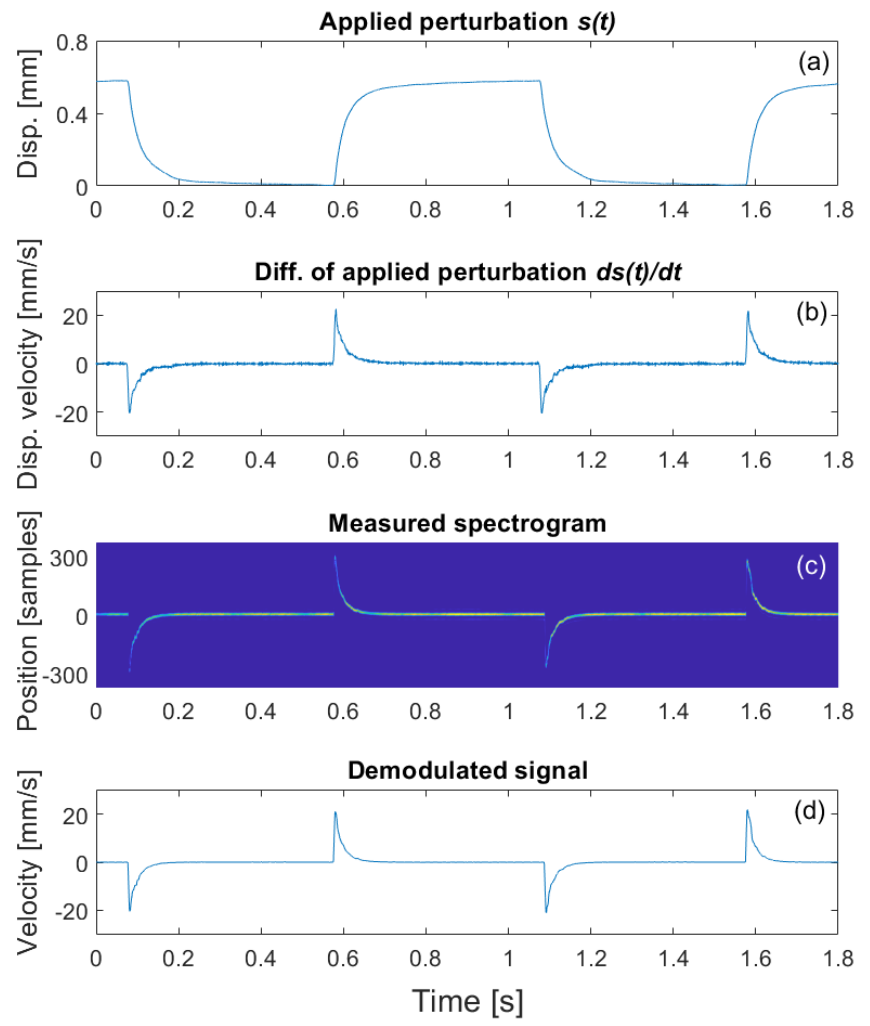

Fig. 8. (a) Displacement induced in the fiber, (b) its derivative with respect to time, (c) spectrogram measured and (d) demodulated signal.

\section{Characterization of the demodulated signal}

A characteristic of this technique is the dependence of the measured amplitude and time response (in samples) on the scanning speed of the TLS $\gamma$. Therefore, this is a key parameter to define the final performance of the system: frequency and spatial resolution/amplitude limits.It is clear in (14) that the amplitude in the detected waveform is influenced by the TLS speed $(\gamma)$ and the optical frequency $\left(f_{0}\right)$. Since the optical frequency variation in the operation range is much smaller than other factors, its influence is neglected in this work.

Figure 9 depicts the demodulated spectrogram of the squarewave shown in Fig.8 (a) for different TLS tuning speeds. It is evidenced that the detected amplitude has an inverse relationship with the tuning speed for the same perturbation. The proper characterization of this relationship is presented in Fig. 10 by measuring the detected amplitude in samples using a sinusoidal signal at $50 \mathrm{~Hz}$ while changing the tuning speed from 10 to $1.3 \mathrm{THz} / \mathrm{s}$. As expected, there is a linear relationship between the measured amplitude and $1 / \gamma$ with a slope of 530.8 samples. THz/s and a fitting error $\mathrm{R}^{2}=0.9999$.
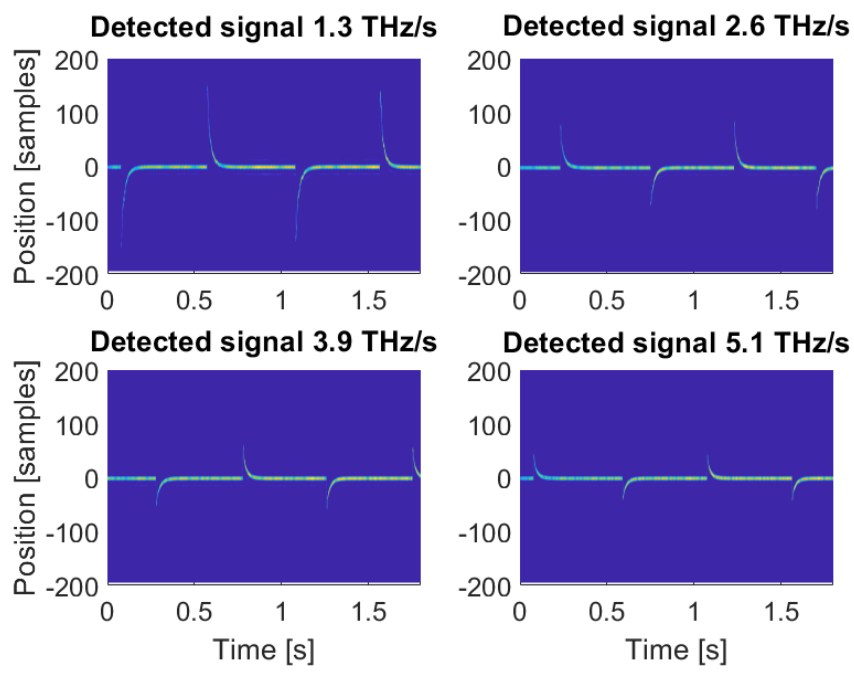

Fig. 9. Spectrograms of a square wave for TLS tuning speeds $\gamma$ of 1.3, 2.6, 3.9 and $5.1 \mathrm{THz} / \mathrm{s}$.

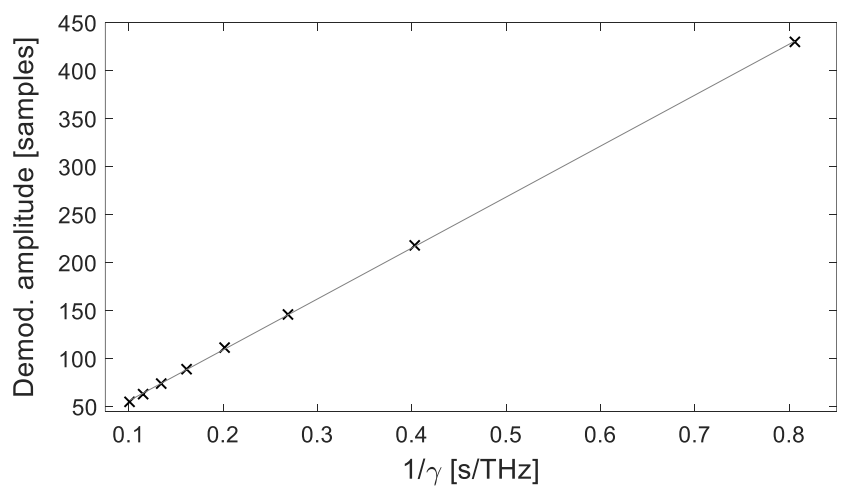

Fig. 10. Relationship of detected amplitude in samples vs $1 / \gamma$ for a $50 \mathrm{~Hz}$ sinusoidal signal. 


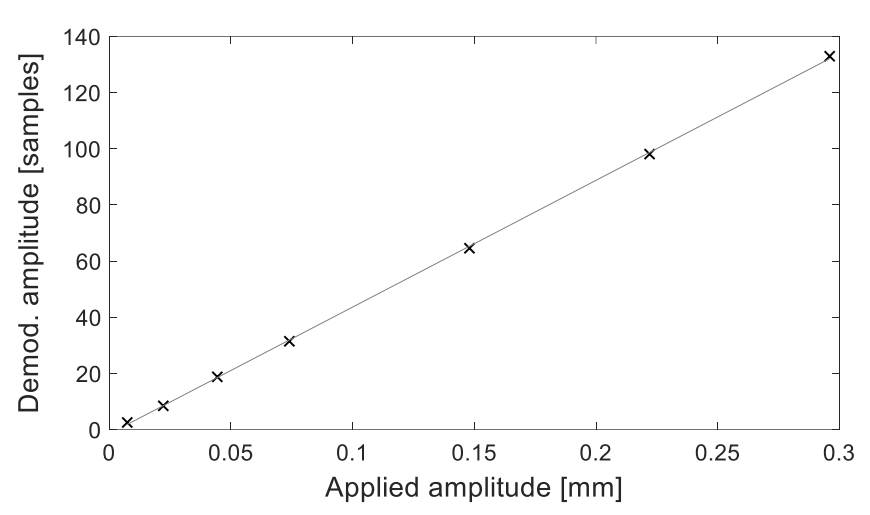

Fig. 11. Dependence of demodulated vibration amplitude vs $D$.

Moreover, the detected signal will also depend linearly on the amplitude of the applied perturbation $D$. To verify the principle, several $50 \mathrm{~Hz}$-sinusoidal waves with different amplitudes are measured keeping the OFDR parameters constant. The results plotted in Fig. 11 evidence the linear relationship with a slope of 451.3 samples $/ \mathrm{mm}$ and $\mathrm{R}^{2}=0.9998$.

Another crucial factor of dynamic strain sensors, velocimeters and accelerometers is the ability to detect a wide range of frequencies. One of the main differences of this proposal compared to other approaches based on dynamic strain rate measurements using OFDR, is that the frequency range is not limited by the repetition rate of the TLS. In this case, the range of detectable frequencies depends on the effective sampling rate, but also on the STFT parameters such as the window shape, window size, and the shift between consecutive window positions. Since STFT is applied for the demodulation, the sampling rate described in (13) is finally down sampled by a factor defined by the window shift between FFT calculations $\left(N_{\text {shift }}\right)$. Consequently, also considering the Nyquist criterion, the maximum detected frequency satisfies $f_{\max }<f_{s} /\left(2 \cdot N_{s h i f t}\right)$.
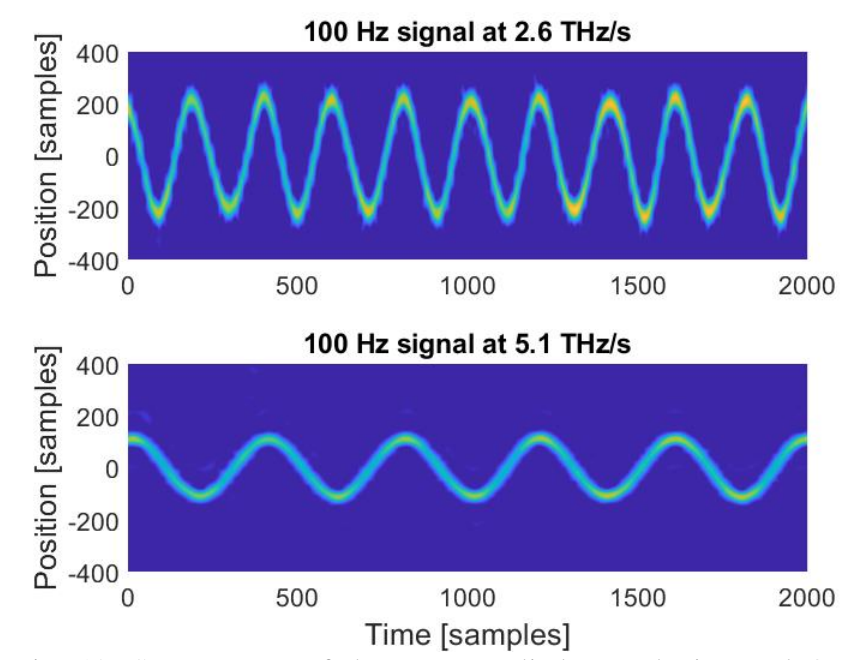

Fig. 12. Spectrogram of the same applied perturbation and OFDR settings except for $\gamma=2.6$ and $5.1 \mathrm{THz} / \mathrm{s}$.
It should be noted that the sampling rate has a linear relationship with the TLS tuning speed $\gamma$. Accordingly, the maximum detected frequency will vary with $\gamma$ as well. Moreover, as commented previously [Fig. 10 and (14)], the detected amplitude is inversely proportional to $\gamma$. These interrelated effects are shown in Fig. 12. For the same applied vibration and demodulation settings, the detected amplitude of the sinusoidal pattern in the spectrogram halved by doubling the TLS speed from 2.6 to $5.1 \mathrm{THz} / \mathrm{s}$; meanwhile, the period of the sine wave is doubled in the view area. Note that in order to compare the behavior, the results are presented in samples.

A series of experiments were carried out to verify the relationship between detected and applied frequency under different TLS configurations. Figure 13 represents the normalized frequency detected (inverse of the number of samples per period) for different vibration frequencies and tuning speeds ranging from 1.3 to $10 \mathrm{THz} / \mathrm{s}$. As expected, there is a linear correlation between detected and applied frequency while this relation depends on the TLS tuning speed. As a consequence, the frequency range that can be detected is directly linked to this relationship. The experiments were performed up to $2 \mathrm{kHz}$; however, it is expected that the system maximum detectable frequency is greater, especially for high TLS speeds. Also, shorter STFT windows would increase this limit, at the cost of a reduced amplitude resolution. An example of the experimental results for a $2 \mathrm{kHz}$ signal at $\gamma=10 \mathrm{THz}$ is presented in Fig. 14, showing an accurate detection of the sinusoidal perturbation.

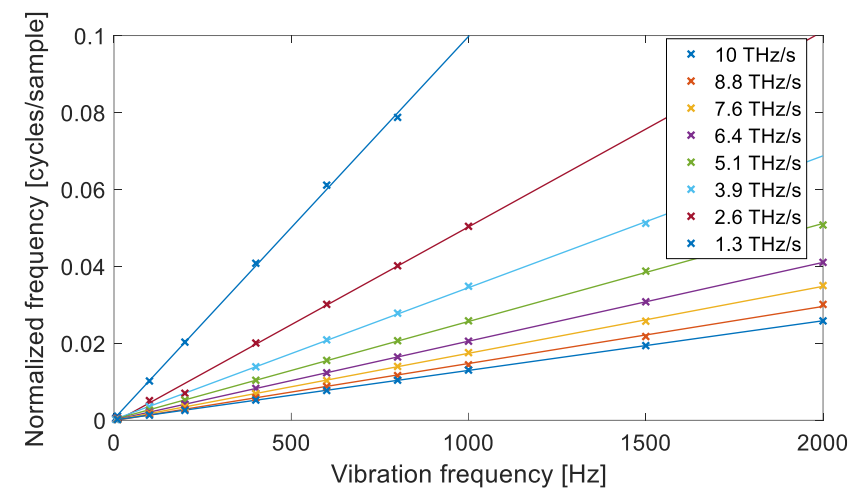

Fig. 13. Detected frequency for different vibration frequencies and tuning speed $\gamma$.

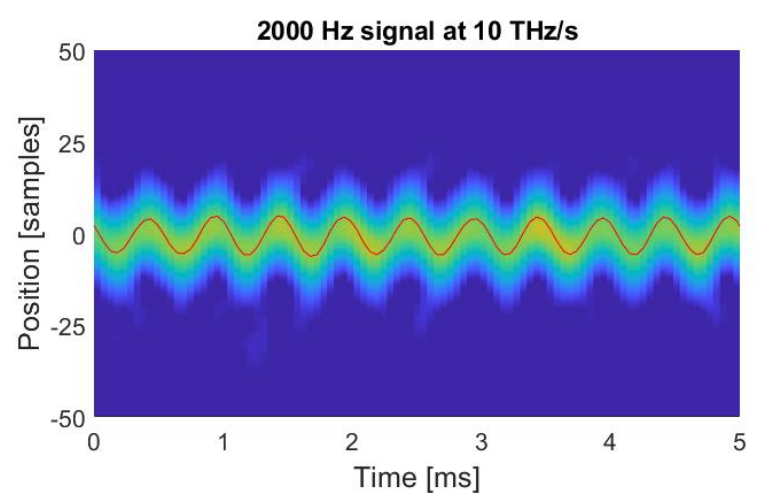

Fig. 14. Spectrogram and demodulated signal for a $2 \mathrm{kHz}$ sinusoidal perturbation at tuning speed $\gamma=10 \mathrm{THz}$. 


\section{DISCUSSION}

The solution proposed is a quasi-distributed velocimeter based on OFDR and an array of weak reflectors. To evaluate the performance of the system, some other aspects such as spatial resolution, crosstalk, or maximum measuring distance should be discussed.

The purpose of the weak reflectors is just to generate a reflection that stands out over the noise floor so the accumulated variation due to the perturbations can be detected without crosstalk induced by the multi-reflection between sections. In practice, the $2^{\text {nd }}$-order signal for a reflectivity $R_{f}$ will be reduced by a $R_{f}^{2}$ factor so the impact of this type of crosstalk is very limited when using a weak reflector. Moreover, due to the detection process based on the STFT, the demodulated signal is highly insensitive to amplitude changes induced by multi-reflection crosstalk.

In this approach, the spatial resolution is defined by the distance between the reflectors. To avoid frequency crosstalk, the phase variations induced by the perturbation must generate a change in the corresponding reflector spectrogram smaller than the distance between segments (so it does not overlap with the next/previous reflector).

It should be noted that using this technique, both spectrogram axes represent two different domains. The distance axis represents also multiple velocity axes, centered at the position of each reflector, of the perturbation happening in the segment right before them. In the same manner, the frequency axis of the spectrogram also represents the time domain. Taking this into account, the maximum detectable velocity without crosstalk defined in (14) is limited by the distance between reflectors, but it can be adapted using $\gamma$. Depending on the application this limitation could become almost negligible with an appropriate election of $\gamma$. For example, in the mechanical characterization of structures, like performing modal analysis. If the deformation is distributed along the fiber, reducing the distance between reflectors reduces in the same manner the total phasechange induced per segment (since the integration length is reduced). Thus, the impact of increasing the spatial resolution is very limited in some applications. Taking all this into consideration, an adequate adjustment of the system for the application can easily offer spatial resolutions in the centimeters order.

It is important noting that the laser tuning speed $\gamma$ is a key parameter in the design. Increasing this value reduces the detected amplitude, allowing an improvement of the spatial resolution without crosstalk. On the other hand, it increases the maximum detected frequency at the cost of the corresponding increase in the data load. Also note that due to the velocimeternature of the system, the amplitude is proportional to the instantaneous frequency. This implies that high frequency signals have an increased visibility, compared to vibration sensors based on dynamic strain measurements.

Another parameter of the laser, the wavelength range of the sweep, has in theory little impact on the technique itself as long as the sweep range is linear and wider than the window length of the STFT. In practice, it can limit the measuring time if a single-direction sweep is employed. However, an appropriate design of the reflectometer might achieve quasi-continuous operation. Regarding the chromatic dispersion, it has been found to be negligible for the wavelength ranges and fiber distances of the study.

Regarding the maximum measuring distance, this approach presents the same limitations than any other OFDR system, which is mainly the phase noise of the laser source. Compared to other techniques such as the OTDR-based, this solution is better suited to structures in the order of tens-hundreds of meters due to the higher spatial resolution and linear response. The maximum number of sensing elements is defined by the inline weak reflectors included in the array. Since the reflectivity of each element can be as low as $\sim 10^{-5}$, the number of sensing sections is practically limited by the spatialresolution required to avoid crosstalk in medium-sized setups. An important advantage over other approaches such as [10] is the capability of the system to measure any arbitrary perturbation, not only periodic vibrations. Moreover, this proposal allows measuring high-amplitude signals and retrieves the absolute value of the perturbations without ambiguity (an important downside in Rayleigh-based DVS [15]).

\section{CONCLUSION}

A new system and demodulation technique based on the Doppler effect have been proposed for quasi-distributed vibration sensing. The setup employs a low repetition-rate OFDR and an array of weak reflectors. A demodulation based on the short-time Fourier transform (STFT) is designed to obtain the spectrogram, detect the frequency variation and compensate the accumulated response. In this manner, the perturbations occurring in the fiber segment between neighboring reflectors can be measured accurately. A theoretical development of the approach has been presented and demonstrated, evidencing the velocimeter operation of the setup. The correct performance of the proposal has been evaluated in several tests using a $10 \mathrm{~m}$ standard single-mode fiber under different perturbation conditions. The system is highly configurable by modifying the tuning speed of the tunable laser. The impact of this parameter has been proved to be proportional to the maximum frequency limit and inversely proportional to the detected amplitude. Finally, concepts as spatial resolution, maximum measuring distance, amplitude ranges have been discussed. This solution offers multiple advantages such as the ability to detect arbitrary perturbations, simplicity, high configurability, linear response to the stimulus or the low repetition rate required in the OFDR. These properties can be exploited in multiple applications, such as in aeronautics, the mechanical characterization of structures or structural-health monitoring among others. Further work is being done to evaluate the operation of the system in real applications. 


\section{REFERENCES}

[1] T.M. Daleyet al., "Field testing of fiber-optic distributed acoustic sensing(DAS) for subsurface seismic monitoring," Leading Edge, vol. 32, no. 6,pp. 699-706, 2013.

[2] K. Johannessen and B. K. Drakeley, "Distributed acoustic sensing: A newway of listening to your well/reservoir," SPE Intell. Energy Int., SPE-149602-MS, 2012.

[3] J. C. Juarez, E. W. Maier, K. N. Choi, and H. F. Taylor, "Distributed fiber-optic intrusion sensor system," J. Lightw. Technol., vol. 23, no. 6, pp. 2081-2087, Jun. 2005.

[4] A. Barrias, J. R. Casas and S. Villalba, "A review of distributed optical fiber sensors for civil engineering applications," Sensors, vol. 16, no. 5, pp. 748, 2016.

[5] D. Wada, H. Igawa, M. Tamayama, T. Kasai, H. Arizono, H. Murayama, and K. Shiotsubo, "Flight demonstration of aircraft fuselage and bulkhead monitoring using optical fiber distributed sensing system," Smart Materials and Structures, vol. 27, no. 2, pp.025014, 2018.

[6] D. Wada, H. Igawa and T. Kasai, "Vibration monitoring of a helicopter blade model using the optical fiber distributed strain sensing technique," Appl. Opt. vol. 55, pp. 6953-6959, 2016.

[7] R. Di Sante, "Fibre optic sensors for structural health monitoring of aircraft composite structures: Recent advances and applications," Sensors, vol. 15, no. 8, pp. 18666-18713, 2015.

[8] D. Arbel and A. Eyal, "Dynamic optical frequency domain reflectometry," Opt. Express, vol. 22, pp. 8823-8830, 2014.

[9] A. B. Am, D. Arbel, and A. Eyal, "OFDR with double interrogation for dynamic quasi-distributed sensing," Opt. Express, vol. 22, pp. 22992308, 2014.

[10] M. Zhu, D. Leandro, M. López-Amo and H. Murayama, "Quasidistributed vibration sensing using OFDR and weak reflectors," Opt. Lett., vol. 44, pp. 1884-1887, 2019.

[11] C. Zhou, T. Tian, L. Qian, D. Fan, W. Liang and Y. Ou, "Doppler EffectBased Optical Fiber Vibration Sensor Using Frequency-Shifted Interferometry Demodulation," J. Lightw. Technol., vol. 35, no. 16, pp. 3483-3488, Aug. 2017.

[12] K. Kageyama, H. Murayama, K. Uzawa, I. Ohsawa, M. Kanai, Y. Akematsu, K. Nagata, and T. Ogawa, "Doppler effect in flexible and expandable light waveguide and development of new fiber-optic vibration/acoustic sensor," J. Lightw. Technol., vol. 24, no. 4, pp. 17681775, Apr. 2006.

[13] H. Igawa, K. Ohta, T. Kasai, I. Yamaguchi, H. Murayama, and K. Kageyama, "Distributed measurements with a long gauge FBG sensor using optical frequency domain reflectometry," Journal of Solid Mechanics and Materials Engineering, vol. 2, no. 9, pp. 1242-1252, 2008.

[14] H. Murayama, D. Wada, and H. Igawa, "Structural health monitoring by using fiber-optic distributed strain sensors with high spatial resolution," Photonic Sensors, vol. 3, no. 4, pp. 355-376, 2013.

[15] A.H. Hartog, "Rayleigh backscatter: distributed vibration sensors and static measurements," in An Introduction to Distributed Optical Fibre Sensors, $1^{s t}$ ed. CRC Press, 2017, ch. 6. 